\title{
Investigation of Flexible Walls for Acoustic Liners
}

\author{
Karsten Knobloch, Lars Enghardt† and Friedrich Bake B $^{\ddagger}$ \\ DLR Engine Acoustics, Institute of Propulsion Technology, German Aerospace Center, 10623 Berlin, GERMANY
}

This paper describes the continuation of work on a novel concept aiming at the improvement of acoustic damping characteristics of liner structures. Here, a first attempt to characterize the actual vibrations of the flexible liner structures is presented and the results obtained from different test procedures are compared.

\section{Nomenclature}

\author{
$f \quad$ frequency $[\mathrm{Hz}]$ \\ $m, n \quad$ bending mode orders for flexible elements[-] \\ M Mach number[-] \\ $r \quad$ reflection coefficient (amplitude) [-] \\ $R \quad$ reflection coefficient (energy) [-] \\ $t \quad$ transmission coefficient (amplitude) [-] \\ $T \quad$ transmission coefficient (energy) [-] \\ $\Delta \quad$ dissipation coefficient (energy) [-] \\ Superscript \\ $+\quad$ downstream propagating \\ - $\quad$ upstream propagating \\ Abbreviations \\ SDOF $\quad$ single degree of freedom (liner) \\ SPL sound pressure level $[\mathrm{dB}]$ \\ TPU thermoplastic polyurethane
}

\section{Introduction}

Liners are part of every modern commercial aero-engine. Usually, they are installed in the engine intake; but also in the bypass duct or in the outlet specific liners can be found. Despite the decrease of overall engine noise due to the increase of bypass-ratio (BPR), cut-off design for rotor-stator combinations, and various other means, there is an increasing demand for efficient broad-band noise absorption with the final goal of further engine noise reduction. The expected further increase in BPR requires shorter nacelles in order to reduce associated drag and weight penalties. This leads not necessarily to a smaller area for liner installation e.g. in the intake of the engine, but to a shorter length of the intake and thereby to a shorter propagation distance of emitted noise over a lined surface in axial direction. Besides the afore-mentioned reasons for efficient broadband noise damping, there is also a need for efficient damping at lower frequencies, which is usually limited by the available space (specifically depth) for the liner installation which limits the cell size.

A novel liner concept with an enhanced acoustic damping performance was introduced ${ }^{1}$ which obtains improved acoustic damping by the interaction of the sound field with flexible walls within an otherwise classical liner cavity structure. Hereby, the deflection of the cell walls and intrinsic damping of the wall

\footnotetext{
*Scientist, correspondence to: karsten.knobloch@dlr.de

†Professor at TU Berlin and head of DLR department "Engine Acoustics", AIAA Member

${ }^{\ddagger}$ Scientist, AIAA Member
} 
material is supposed to yield improved damping characteristics in terms of overall absorption (dissipation) of acoustic energy and an increase of the frequency range of significant absorption. This concept is called "FlexiS" (Flexible Structure) liner. The FlexiS liner utilized in earlier investigations was designed to be tested in the aerocoustic DUCT windtunnel under plane wave excitation. The setup of the protoype for the wind-tunnel measurements is shown in Fig. 1.

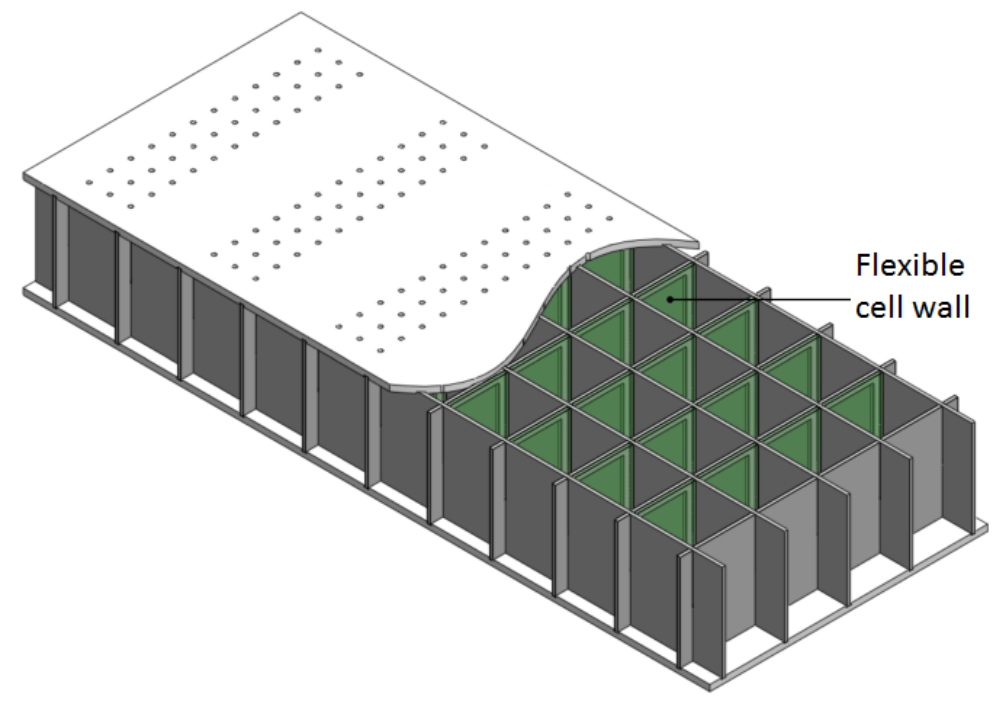

Figure 1. FlexiS liner concept with partially flexible walls in the liner cavity structure.

Aero-acoustic measurements showed the dependence of the acoustic dissipation obtained by this concept on material and thickness of the flexible wall material (Figure 2).

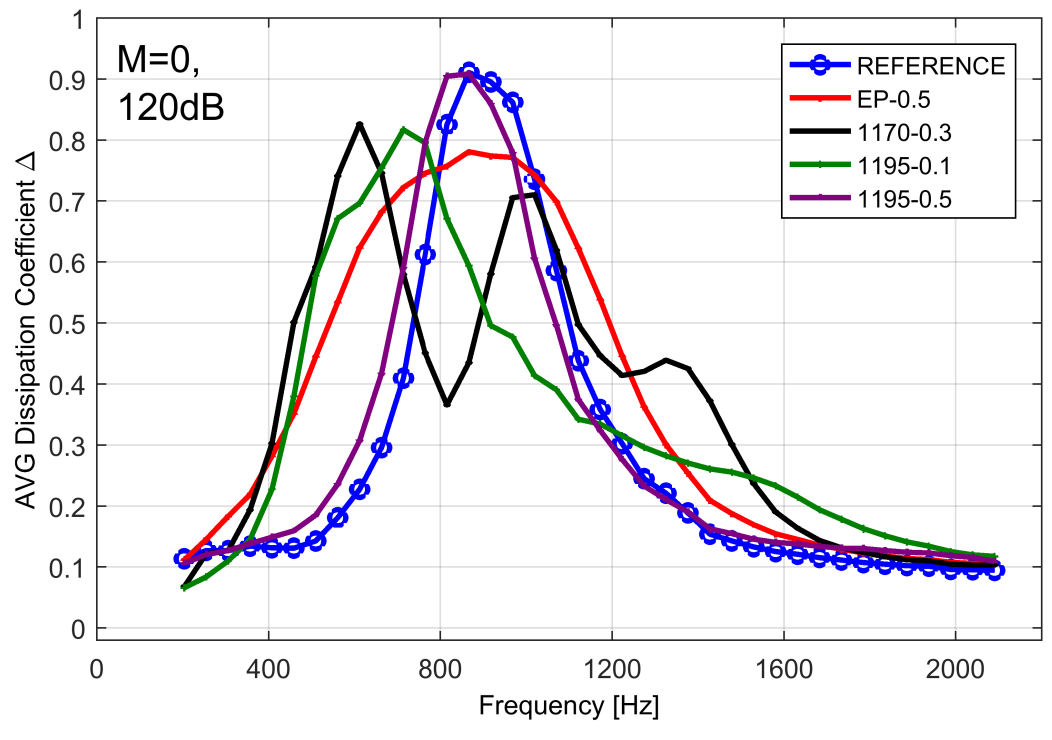

Figure 2. Averaged dissipation of acoustic energy for FlexiS liner.

Compared to the "REFERENCE" liner (a sample build in the same way as the FlexiS liner, but with rigid cell walls) most of the liners with flexible materials demonstrate a significant increase in the dissipation in the low frequency range from 400 to $800 \mathrm{~Hz}$. While the EP-05 material generates a broadening of the dissipation peak, the material 1170-03 exhibits three dissipation maxima around 600, 1000, and $1350 \mathrm{~Hz}$. For the sample with the material 1195-01 the dissipation maximum is shifted from $940 \mathrm{~Hz}$ (REFERENCE) to around $750 \mathrm{~Hz}$. Most FlexiS liner samples showed a reduction of the maximum dissipation value (exception 
1195-05). However, the integrated overall dissipation over the entire frequency range is increased. This verifies the additional damping mechanism by the deflection of the flexible walls with the intrinsic material damping characteristics. The change in acoustic dissipation - i.e. the broadening of the acoustic dissipation frequency range, and in part also a shift towards lower frequencies - could be also observed for tests with a grazing flow $(\mathrm{M}=0.1$ and $\mathrm{M}=0.2$; not shown here).

\section{Scope of the Current Investigation}

So far, little information could be obtained from the windtunnel measurements about the actual processes in the cells of the liner: How is the flexible wall deflected? Which mode is excited and what are the amplitudes of velocity or displacement? Detailed knowledge is needed for the assessment of the additional damping effect and the modeling of the novel concept. The only information, which could be obtained so far, stem from flush mounted microphone in the lower cell walls - proving the flexible cell wall deflection via the analysis of sound pressure level and phase difference between adjacent cells. ${ }^{1}$

In a new series of experiments, an attempt was made to assess the deflection of the flexible walls in detail. A liner wall mock-up containing four flexible segments (each constitutes the flexible cell wall of a single liner cell) was manufactured and tested when exposed to normal (i.e. perpendicular) acoustic excitation without any flow present. A set of these devices - below referred to as "liner wall samples" - has been built and tested.

As during the previous experiments, different materials were used as test objects - since the material type and thickness did determine the change in acoustic damping with a clear distinction between the results for the different materials. Two commercially available materials were tested, both thermoplastic polyurethanes (TPUs): $1170 \mathrm{~A}$ with a thickness of $0.3 \mathrm{~mm}$ and $1195 \mathrm{~A}$ with a thickness of $0.1 \mathrm{~mm}$ and a thickness of $0.5 \mathrm{~mm}$. In addition, a liner wall sample carrying a custom made-material based on epoxy-resin (EP-0.5) was tested.

The liner wall sample is shown in Figure 3. Each liner wall sample contained four flexible elements of the same material type and thickness.

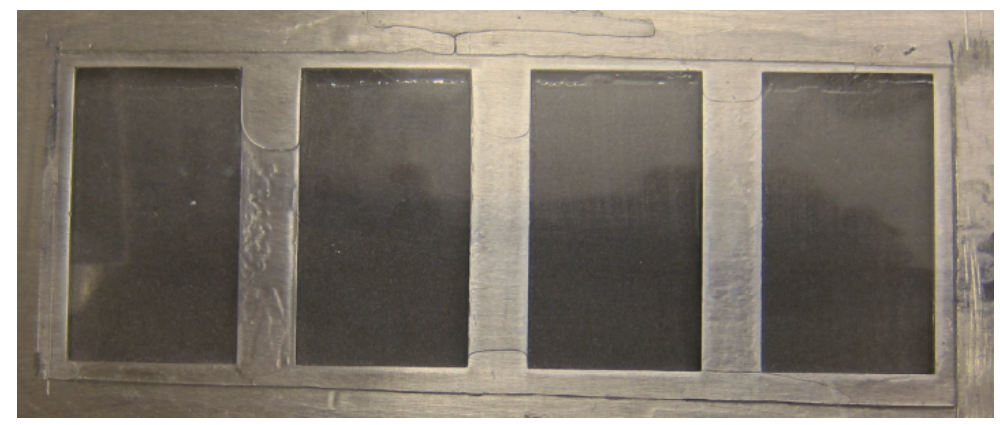

Figure 3. Test object: Liner wall sample with four flexible cell walls used for the present investigation.

\section{Material Description}

The material for the flexible walls needs to exhibit intrinsic (material inherent) damping. This enables the structure to dissipate additional acoustic energy when the flexible walls are deflected. Thereby it constitutes an additional dissipation mechanism for acoustic energy besides the energy conversion taking place at the cavity face sheet of the regular liner.

The materials selected for the initial investigation were chosen based on material properties, availability, and ease of application. Thermoplastic-polyurethan (TPU) materials are easily available as sheets in various thicknesses ranging from one tenth of a millimeter to several millimeters. Material parameters can be obtained from material data sheets (e.g. from $\mathrm{BASF}^{2}$ ) or determined with specific instruments. The dynamic mechanical analysis (DMA) yields the phase lag between stress and strain for a small torsional (or otherwise) deflection. The tangent of this phase lag is called the loss factor $\tan (\delta)$ or $\tan (\Delta)$ which describes the material inherent damping ( $\Delta$ not to be confused with the dissipation of acoustic energy as used throughout this paper). For the materials considered here - TPU sheets of type 1195A in thickness $0.1 \mathrm{~mm}$ and $0.5 \mathrm{~mm}$, 
and type $1170 \mathrm{~A}$ in thickness $0.3 \mathrm{~mm}$ - the loss factor is in the range of 0.05 to 0.3 . Generally, the loss factor is strongly depending on temperature and and to a certain extent also on the frequency of deflection.

A custom made material composed of epoxy resin in combination with polyetheramine was chosen and manufactured with a sheet thickness of $0.5 \mathrm{~mm}$ with the focus on a higher loss factor compared to the commercially available TPU sheets. It is identified in the results section as "EP-05" and exhibits a loss factor of about 1.0 .

\section{Experimental Setup}

In a first setup, the liner wall sample was mounted perpendicular to the mean flow between the two microphone sections of the DUCT-R windtunnel, which was used before for the investigation of the complete liner sample. ${ }^{1}$ Utilizing the regular measurement procedure for liner investigations, reflection, transmission, and dissipation of the liner wall element could be determined.

In a second series of experiments, the liner wall sample was mounted to the upstream section of the duct. A laser-based vibrometer was used to scan deflection and vibration velocity of different points on one of the flexible wall elements of each test object.

\section{Experimental Setup at DUCT Wind Tunnel - Dissipation and Transmission Under Normal Incidence}

The basic setup for the acoustic measurements is identical to the setup for a conventional HelmholtzResonator liner (SDOF) liner. By the upstream and downstream speakers, a multitone is excited and fed into the rig. Consecutively, six multi-tones covering the frequency range from 204 to $2091 \mathrm{~Hz}$ with a $51 \mathrm{~Hz}$ resolution are used. The overall SPL of these multi-tones was adjusted to about 120dB. The cut-on frequency for higher-order modes in the DUCT-R rig is about $2150 \mathrm{~Hz}$ for the no-flow case.

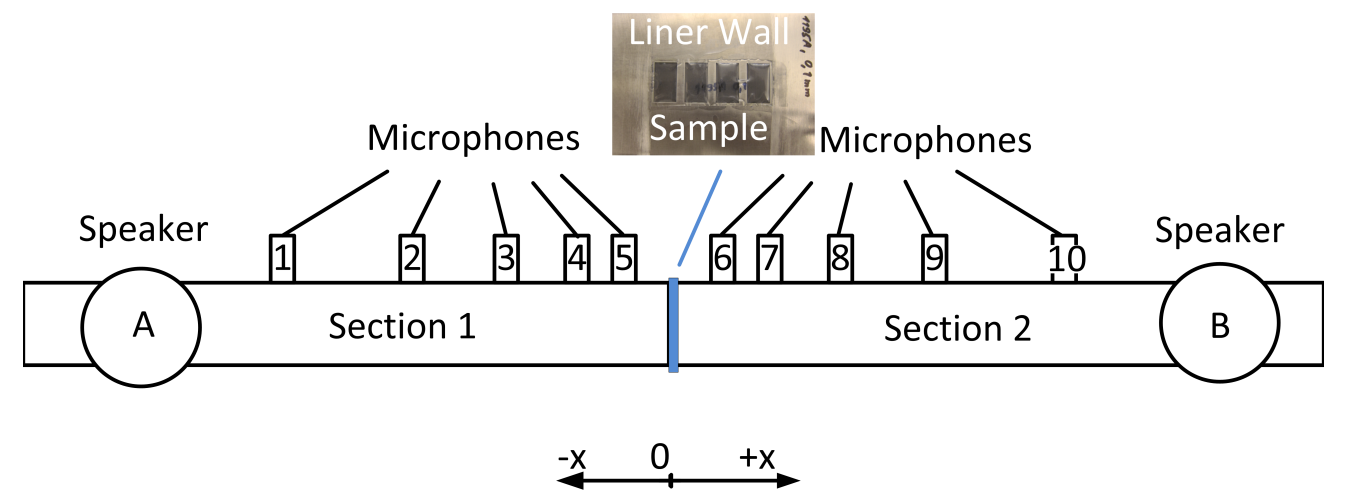

Figure 4. Setup-1: Liner wall sample mounted between both section. Normal incidence of sound field. Measurement of reflection, transmission and dissipation of the overall sample. Anechoic terminations at both ends are not shown in the sketch.

Here, instead of the damping module with the liner, the liner wall sample was mounted between the two microphone segments (depicted in Figure 4). This yields a normal incidence of the propagating sound wave for the flexible wall elements. Only no-flow measurements can be made in this arrangement.

\section{Setup for Deflection Measurements}

The setup for the detailed deflection measurements used mostly the same setup, with section 2 not required here (Figure 5). The liner wall sample was attached to the end of the 60 by $80 \mathrm{~mm}^{2}$ duct section. Five microphones were distributed along the upstream section, with the closest microphone (Mic-5) having a $100 \mathrm{~mm}$ separation from the liner wall sample.

A sweep sine signal $(200-2500 \mathrm{~Hz}$, sweep duration of 1s) was used to acoustically excite the test object. The vibrometer was a Polytec OFV-5000 system with a OFV-200 optical head. Transducer for velocity and displacement are build-in. For the measurements, the largest range for the velocity had to be chosen, which is $50(\mathrm{~mm} / \mathrm{s}) / \mathrm{V}$. Time-traces of $60 \mathrm{~s}$ duration were recorded, capturing also the reference microphone signal. 


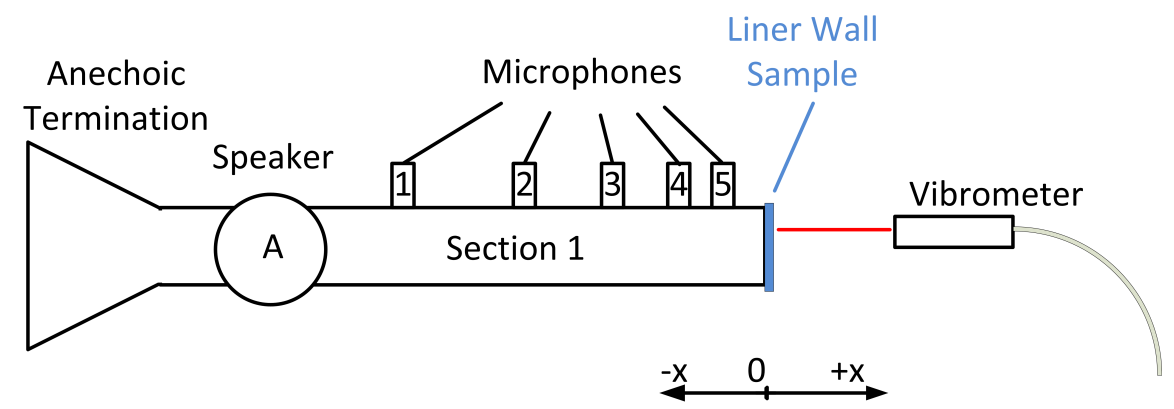

Figure 5. Setup-2: Liner wall sample mounted at the termination of section 1. Normal incidence of sound field. Measurement of local deflection and vibration velocity by vibrometer.

15 points were marked on one of the flexible elements (yielding a spacing of about $5 \mathrm{~mm}$ between markings) which should give a sufficient resolution of at least the first transverse mode in each direction.

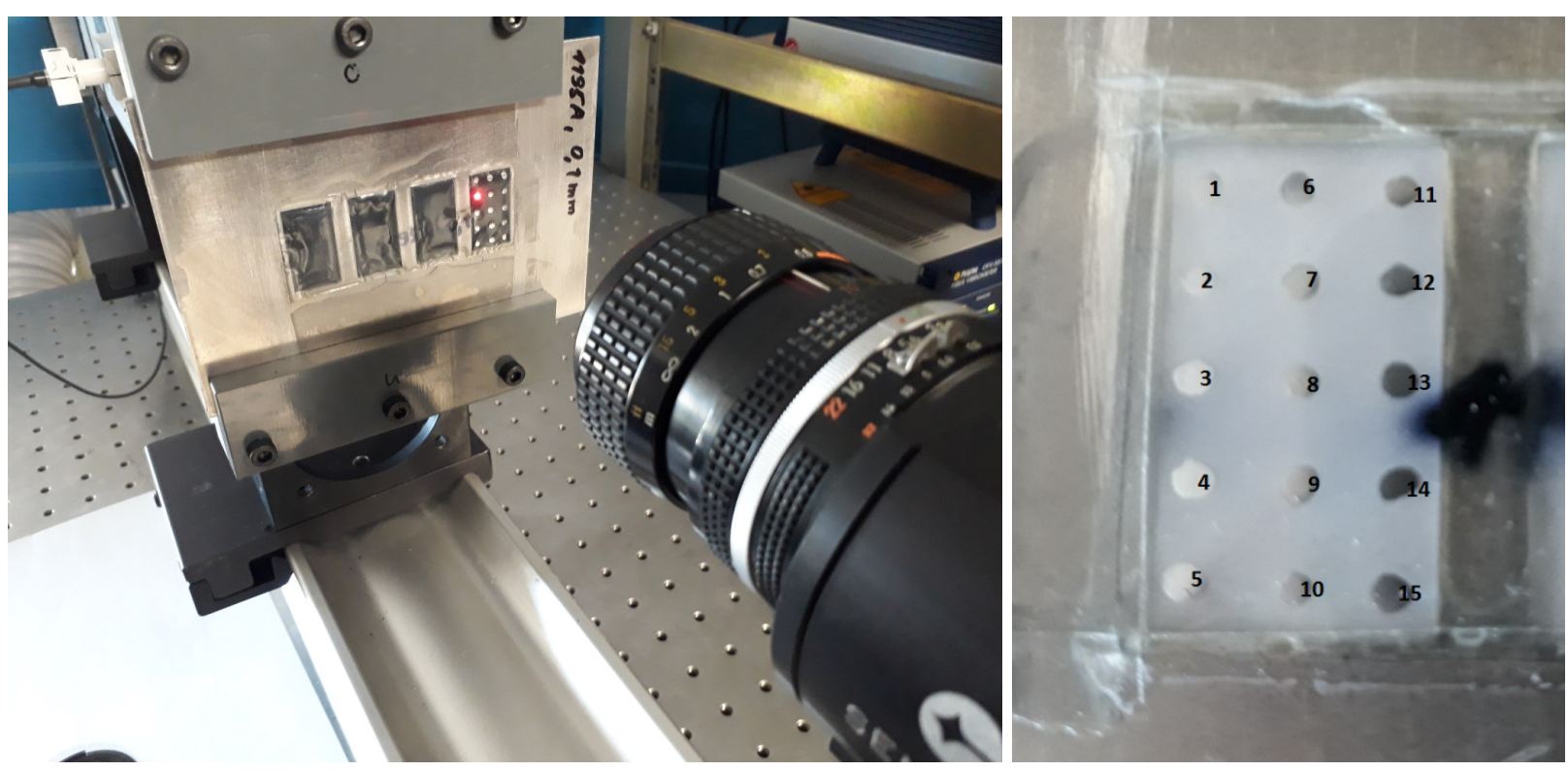

Figure 6. Left: setup with vibrometer, right: 15 markings on one of the flexible elements of the liner wall sample.

\section{Data Processing for Setup-1}

The data processing was performed as described in detail in the previous paper. ${ }^{1}$ The dissipation of acoustic energy is expressed by the dissipation coefficient. The dissipation coefficient can be calculated directly from the reflection and transmission coefficients via an energy balance:

$$
R^{ \pm}+T^{ \pm}+\Delta^{ \pm}=1
$$

For the no-flow case, the dissipation reduces to

$$
\Delta^{ \pm}=1-\left(\left|r^{ \pm}\right|^{2}+\left|t^{ \pm}\right|^{2}\right)
$$

$R$ and $T$ are the power quantities of the reflection and transmission coefficients, while $r$ and $t$ are the pressure quantities.

The dissipation coefficient is an integral quantity used to evaluate the damping performance of a liner. Here, the averaged value $\Delta_{\text {avg }}=\Delta^{+} / 2+\Delta^{-} / 2$ is used to characterize the loss of acoustic energy that is caused by the presence of the liner wall samples. A second quantity to be considered is the transmission, assuming 
that a vibrating section of the insert increases the transmission of acoustic energy for the frequency of vibration.

\section{Data Processing for Setup-2 (Vibrometer)}

The vibrometer yields an analog output signal for the velocity and the displacement/deflection. Both signals were recorded in parallel with the microphone signals with a sampling frequency of $12.8 \mathrm{kHz}$. Subsequent data processing involved several steps:

- Wave decomposition and determination of incident wave

- Determination of transfer function between incident wave and velocity signal

- Identification of local maxima of the transfer function

- Retrival of spatial information for all measurement locations for selected frequencies

The velocity of the vibration was chosen for the evaluation and presentation of data. Assuming a purely sinusoidal deflection of the vibrating structure, the amplitude of the velocity signal is related to the amplitude of the displacement signal by a factor of $2 \pi f$. This implies, that for higher vibration frequencies, the output signal of the vibrometer electronics is larger compared to the output signal of the displacement. In addition, the deflection often decreases with increasing frequency due to decreasing excitation amplitudes. These effects suggest the selection of vibration velocity as the quantity characterizing the vibration.

The different steps for the data processing are illustrated below in the "Results"-section.

\section{Results}

In the following sections, results are first presented for the "two-section" setup determining reflection, transmission, and dissipation. In the second part, results for the vibrometer measurements are shown including intermediate results which demonstrate the data processing steps. A comparison of results in terms of frequencies of identified local maxima is presented at the end of this section.

\section{Results for Acoustic Dissipation Measurements (Setup-1)}

The results are shown in Figure 7. Dissipation maxima found here should directly relate to material-inherent damping, as no other mechanism encountered for the actual liners (cavity resonances, vortex shedding, etc) are present. The material-inherent damping should be most effective, if the structure is vibrating. Furthermore, it can be assumed that the acoustic energy from one side propagates best to the other side of the facility, if the structure is deflected/vibrating. Therefore, an increase in transmission (red curve) correlates with a vibrating flexible structure.

Comparing the transmission and dissipation curves from above figures with the dissipation curves of the complete liner assembly (see Figure 2), a coincidence of effective acoustic damping and the flexible structure vibration can be observed at least for some of the dissipation peaks. A list compiling the frequencies of (local) maxima is given below in Table 1. 

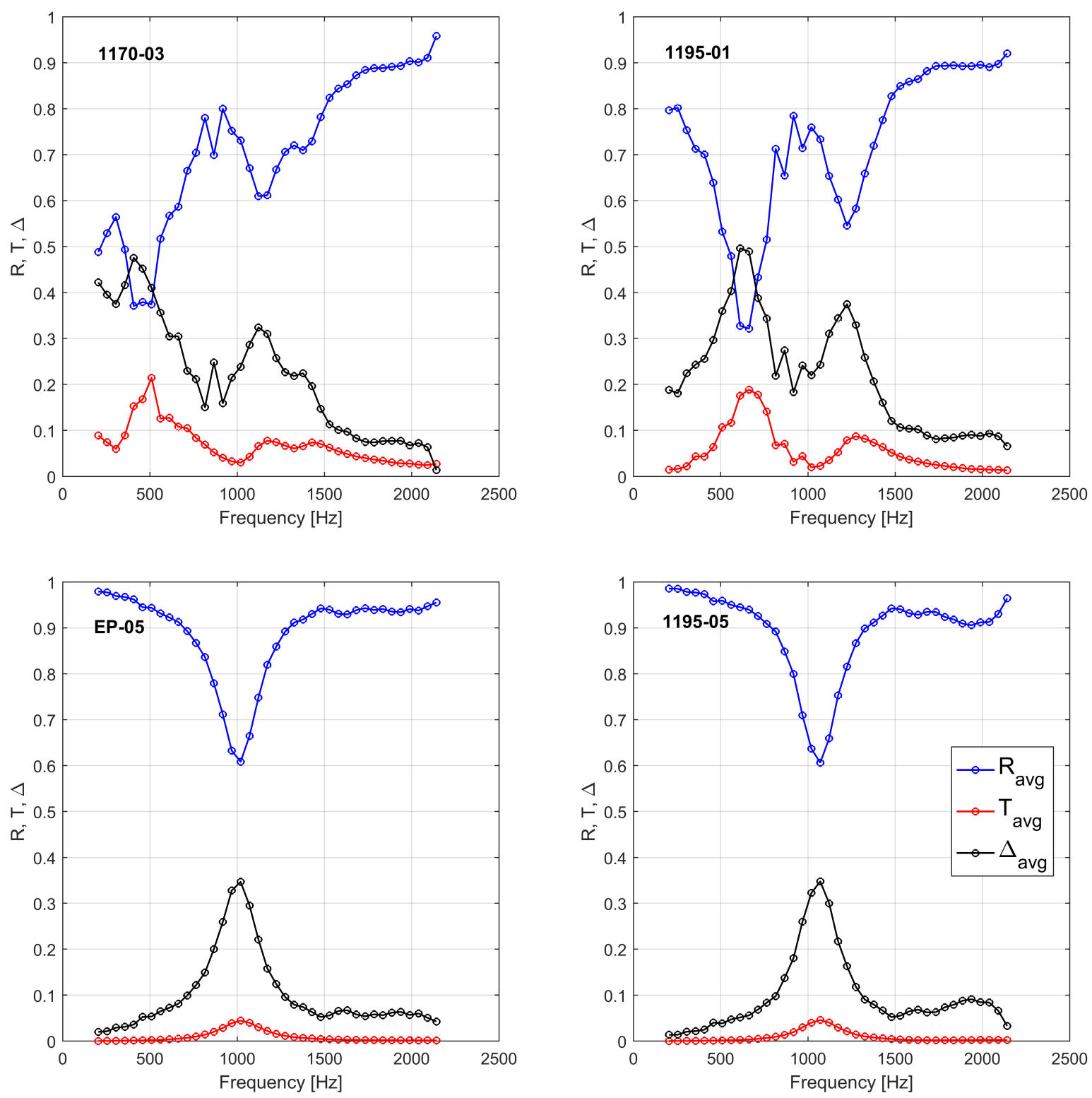

Figure 7. Dissipation $\Delta$, reflection $R$, and transmission $T$ of acoustic energy as determined in the DUCT-R windtunnel for normal incidence. 


\section{Results for Vibrometer Measurements (Setup-2)}

\section{Acoustic excitation in the upstream section}

In order to assess and compare the measured deflection and vibration velocity at the flexible elements, the acoustic excitation needs to be quantified. In Figure 8, the results of the wave decomposition into a downstream traveling wave $p^{+}$and an upstream traveling wave $p^{-}$are shown. Furthermore, the sound pressure level at the microphone closest to the liner wall sample ("Mic-5") is shown. The superposition of upstream and downstream traveling waves yields a destructive interference for a frequency of about $850 \mathrm{~Hz}$, implying, that the microphone signal can not be used as a reliable reference. Instead, the incident wave $p^{+}$ was chosen. The variations with frequency are much smaller than for the individual mcirophone. However, in order to compare results for different frequencies, the transfer function between $p^{+}$and the velocity signal of the vibration is used in the subsequent steps.

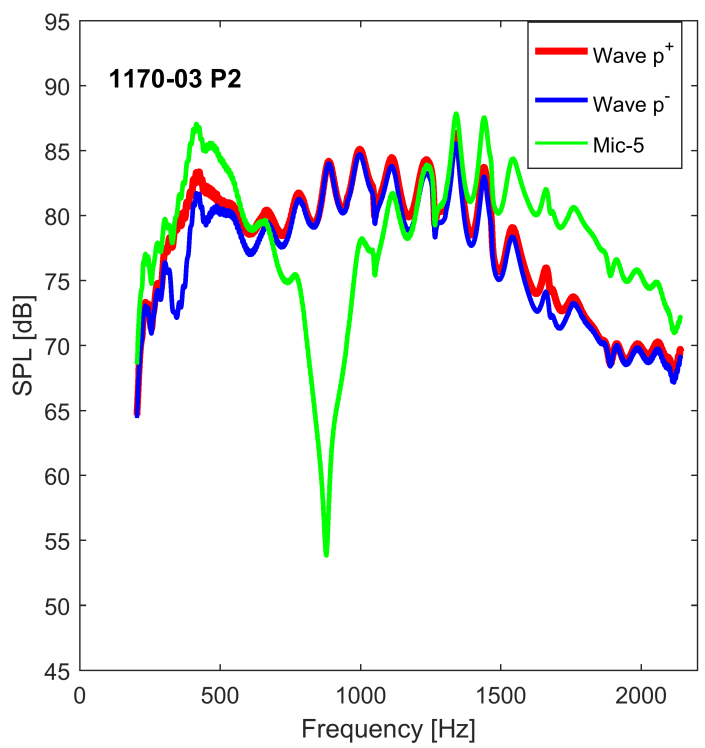

Figure 8. Wave amplitudes calculated in upstream section.

\section{Obtaining Spatial Information}

For each of the 15 points on one of the vibrating segments, the transfer functions $\operatorname{TF}\left(v_{v i b} / p^{+}\right)$was calculated. It is expected, that deflections (and vibration velocities) are largest in the centre of the flexible section, i.e. for point P8 (see Figure 6, right hand side for position of points P1-P15). Thus, higher transverse modes could be hidden, when searching only for maxima of the transfer function in point P8. Therefore, the transfer function for points P2, P6, and P7 were inspected and local maxima identified. For some of the identified frequencies, local maxima are present for all 3 spatial points. In some cases, a significant correlation was found only for one or two of the spatial points.

This procedure is illustrated in Figure 9. Comparing the plots for point P6 (upper right) and point P7 (lower left) it can be observed, that the ratio between local peaks changes drastically, suggesting different spatial modes of vibration. Based on the information of these plots, the frequencies 378, 635, 1161, 1310, and $1650 \mathrm{~Hz}$ were chosen for the sample with material 1170-03 for the collection of transfer function values for all spatial points and the generation of a $2-\mathrm{D}$ plot.

In the upper left graph, the transfer function calculated with microphone 5 is shown too. The example illustrates that a misleading peak of the transfer function is encountered. The transfer functions $\mathrm{TF}\left(x_{v i b}, p^{+}\right)$ between the measured displacement $x_{v i b}$ and the incident wave $p^{+}$are plotted with blue lines. As already described in the "Data Processing" section, the identification of relevant frequencies of vibration from these curves is much more complicated than for the transfer function calculated with the vibration velocity. 

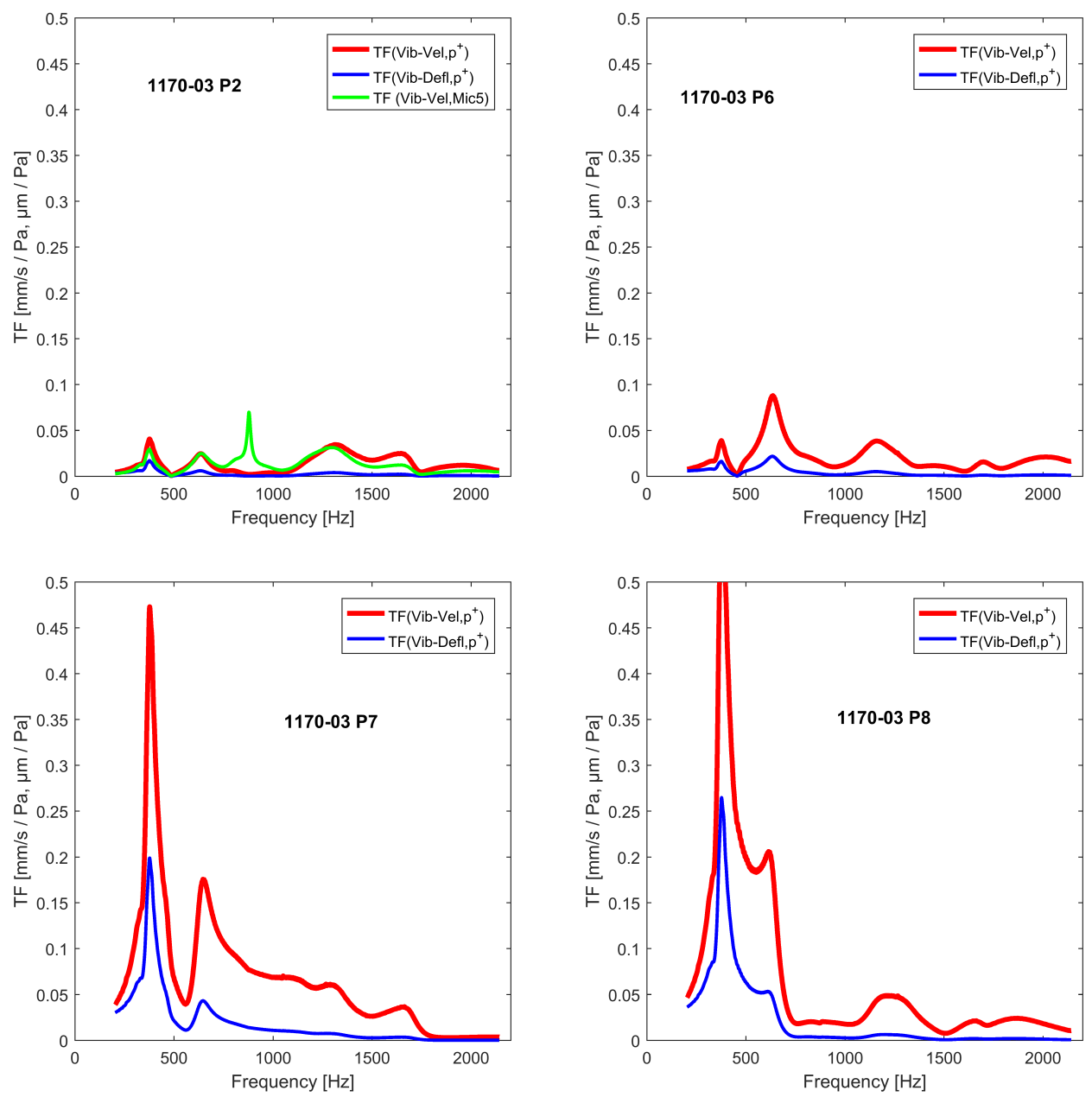

Figure 9. Transfer function vibration velocity/wave amplitude $p^{+}$for 1170-03, points P2,P6,P7,P8.
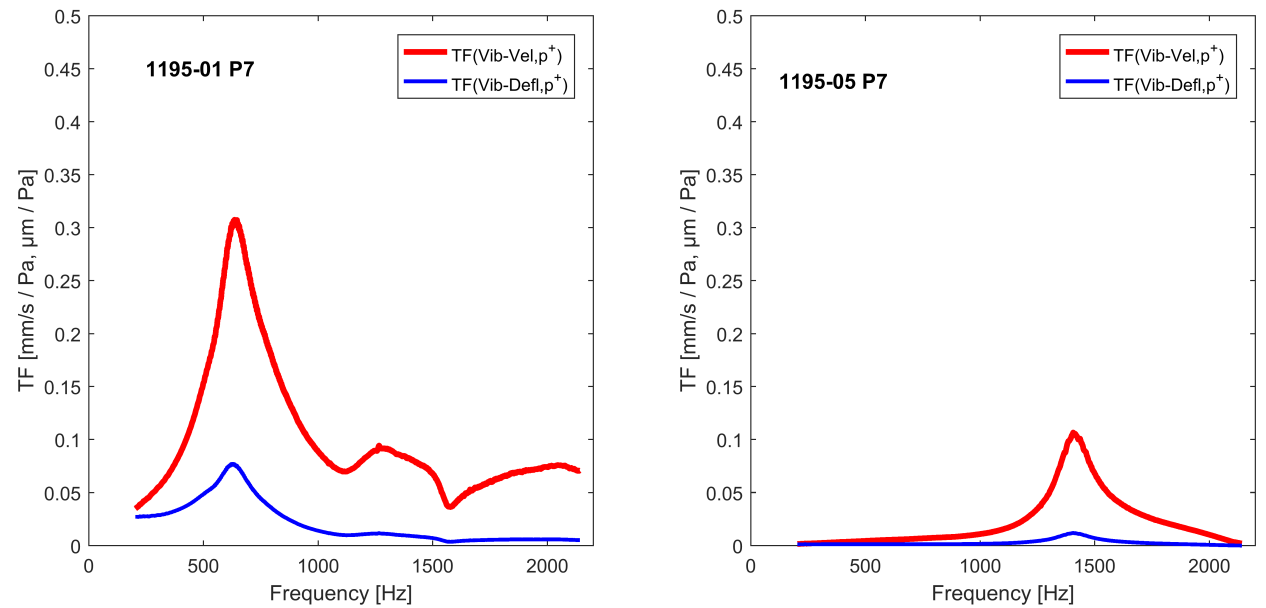

Figure 10. Transfer function vibration velocity/wave amplitude $p^{+}$for 1195-01 and 1195-05, point P7. 
Figure 10 shows the transfer function for point P7 of material 1195 of thickness $0.1 \mathrm{~mm}$ (left) and $0.5 \mathrm{~mm}$ (right). Recalling that the acoustic excitation is always identical, the decrease in deflection velocity, but also the reduction of number of frequencies that are excited, becomes obvious.

\section{Results for Vibrometer Measurements}

Below, the transfer function for frequencies derived from the plots for 1170-03 (as depicted in Figure 9) are shown. For each frequency, a 2-D plot was generated based on the 15 values obtained for P1-P15,
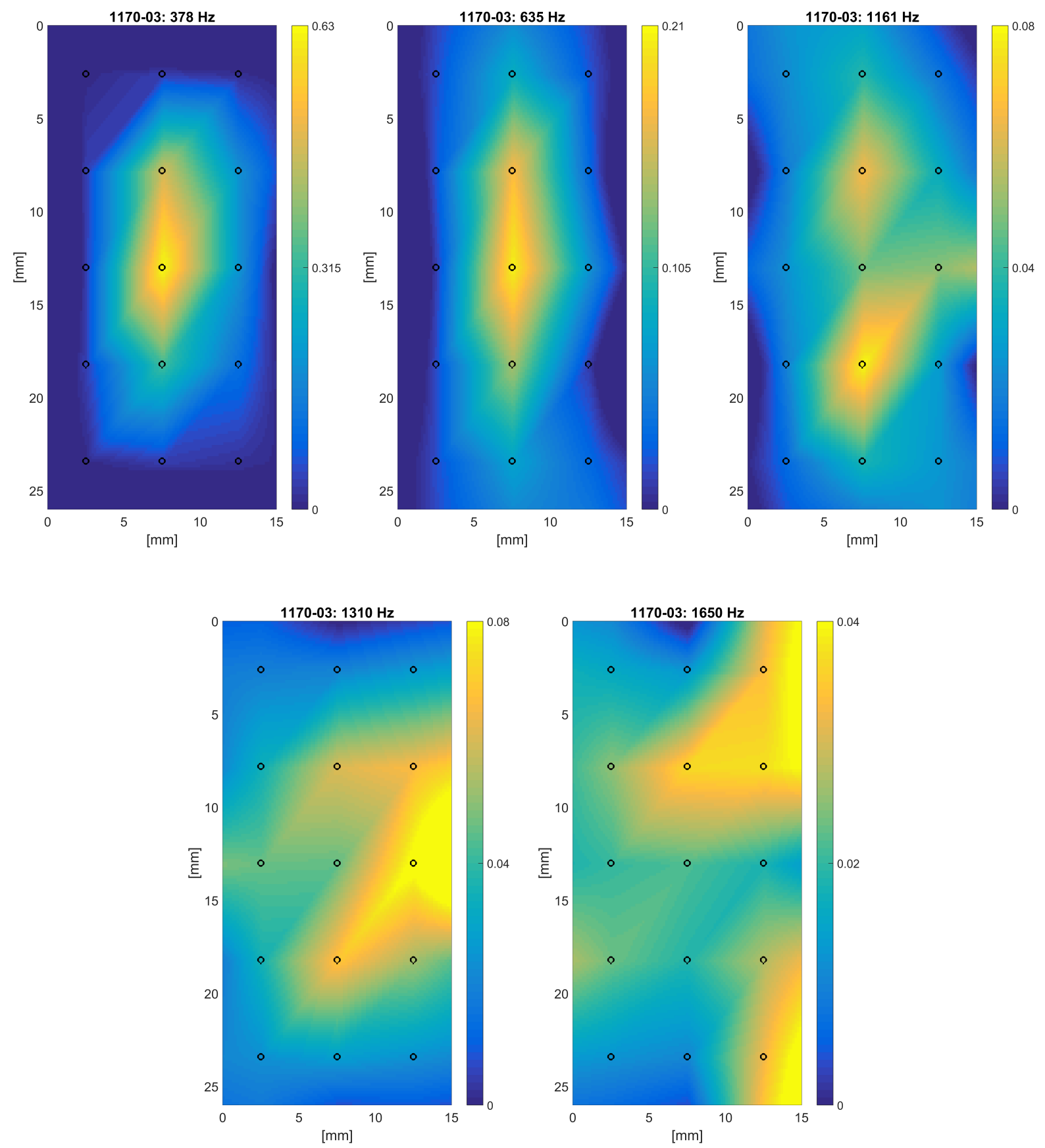

Figure 11. Transfer function between incident wave and vibrometer reading (velocity/deflection signal) for 1170-03 material: $378 \mathrm{~Hz}, 635 \mathrm{~Hz}, 1161 \mathrm{~Hz}, 1310 \mathrm{~Hz}, 1650 \mathrm{~Hz}$. 
subsequently inter- and extra-polating the data on the full area of the flexible material. It should be noted, that this extrapolation procedure may yield some arbitrary values especially at the edges of the considered area.

Figure 11 contains 5 frequencies identified in the transfer function $\operatorname{TF}\left(v_{v i b}, p^{+}\right)$as major peaks or at least "bumps" which have higher values than their neighbouring frequencies. The lower frequencies $378 \mathrm{~Hz}$ and $635 \mathrm{~Hz}$ suggest a fundamental oscillation (bending mode $\mathrm{m}, \mathrm{n}=1$ ). From the low count of spatial points it can not be clearly derived, whether there are actual differences between the vibration modes of these two frequencies (which could be anticipated comparing the more elongated structure for $635 \mathrm{~Hz}$ to the oval shape of $378 \mathrm{~Hz}$ ). The frequency of this bending mode compares well with the observed local maximum in the dissipation curve of the liner (Figure 2: $612 \mathrm{~Hz}$ ). For the intermediate frequency $1161 \mathrm{~Hz}$, a line of vibration nodes crossing P3, P8, and P13 can be anticipated, which suggests a first higher order bending mode. The second dissipation peak of the liner around $1000 \mathrm{~Hz}$ agrees only roughly with this frequency, but should be correlated (considering the clear deviation from peak value near $900 \mathrm{~Hz}$ for the cells of the reference liner). For the higher frequencies $1310 \mathrm{~Hz}$ and $1650 \mathrm{~Hz}$, no clear statement can be made with respect to a possible mode shape. The values for the transfer function are rather low (note different scalings at the color bar, which were chosen to emphasize the modes shapes rather than comparing absolute values). For the lower two plots, the extra-polation towards the edges provides misleading impression regarding the actual mode shape. However, the frequency of $1310 \mathrm{~Hz}$ agrees again with the third maximum with the dissipation curve.

It should be noted: A perfect agreement between dissipation curve peaks and results of the liner cell wall investigation can not be expected due to material and manufacturing tolerances and different boundary conditions inside the liner cavity structure and the windtunnel environment.
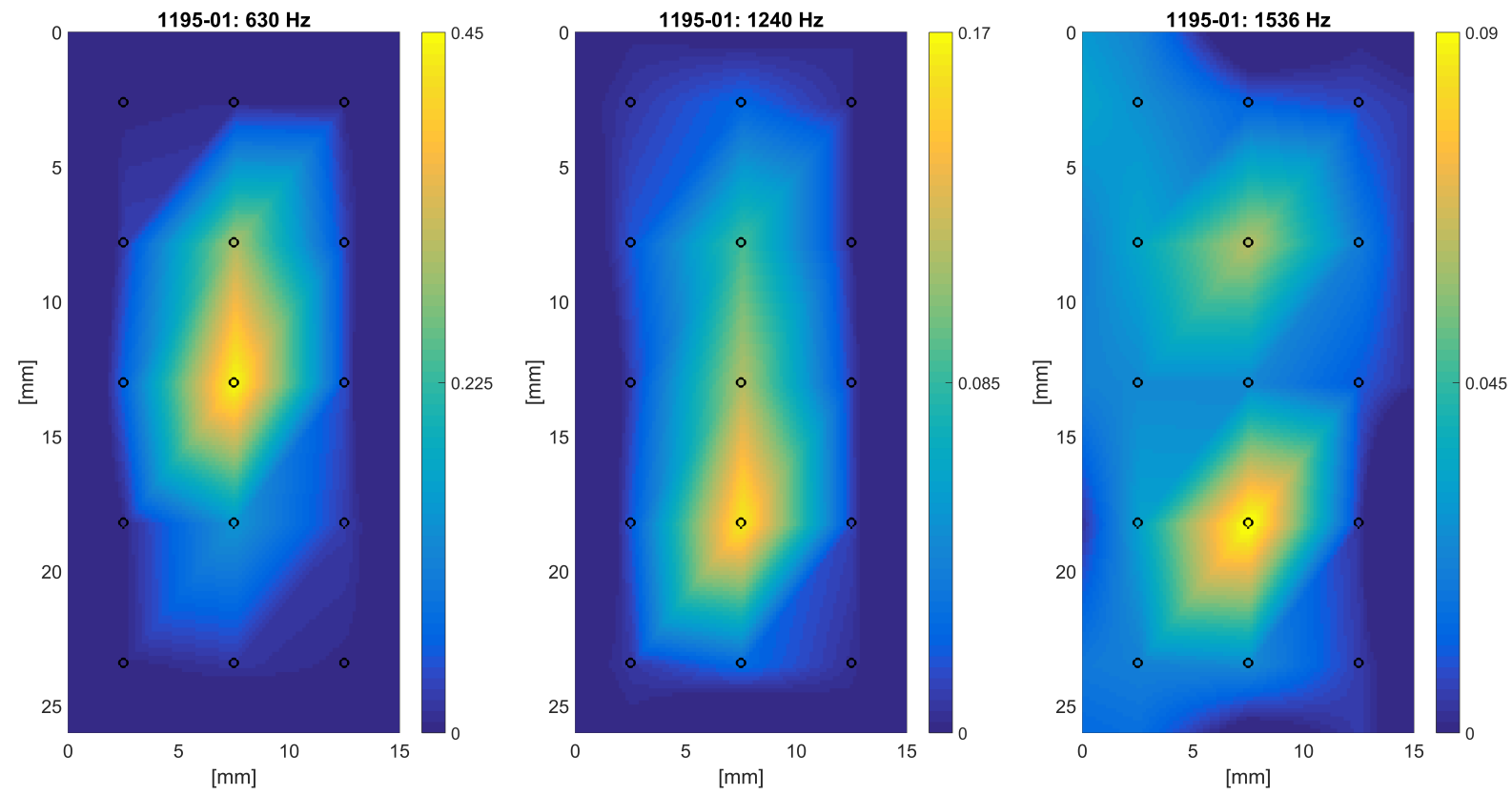

Figure 12. Transfer function between incident wave and vibrometer reading (velocity/deflection signal) for 1195-01 material: $630 \mathrm{~Hz}, 1240 \mathrm{~Hz}, 1536 \mathrm{~Hz}$.

Figure 12 shows the 2-D plots of the transfer function for material 1195-01. As for the material 1170-03, there seems to be a fundamental bending mode for $630 \mathrm{~Hz}$ and a higher order mode for $1536 \mathrm{~Hz}$. The plot for $1240 \mathrm{~Hz}$ exhibits some asymmetrie, which can not be further explained with the current measurements. While the peak at $630 \mathrm{~Hz}$ closely coincides with the major peak in dissipation for the liner structure at $714 \mathrm{~Hz}$ (Figure 2), the absolute values of the vibration velocity (and thereby the transfer function) are smaller for $1536 \mathrm{~Hz}$. Close examination of the dissipation curve reveals a small "bump" in the otherwise decreasing dissipation values with increasing frequency.

For the other two samples, 1195-05 and EP-05, the transfer functions are shown in Figure 13. Again, 

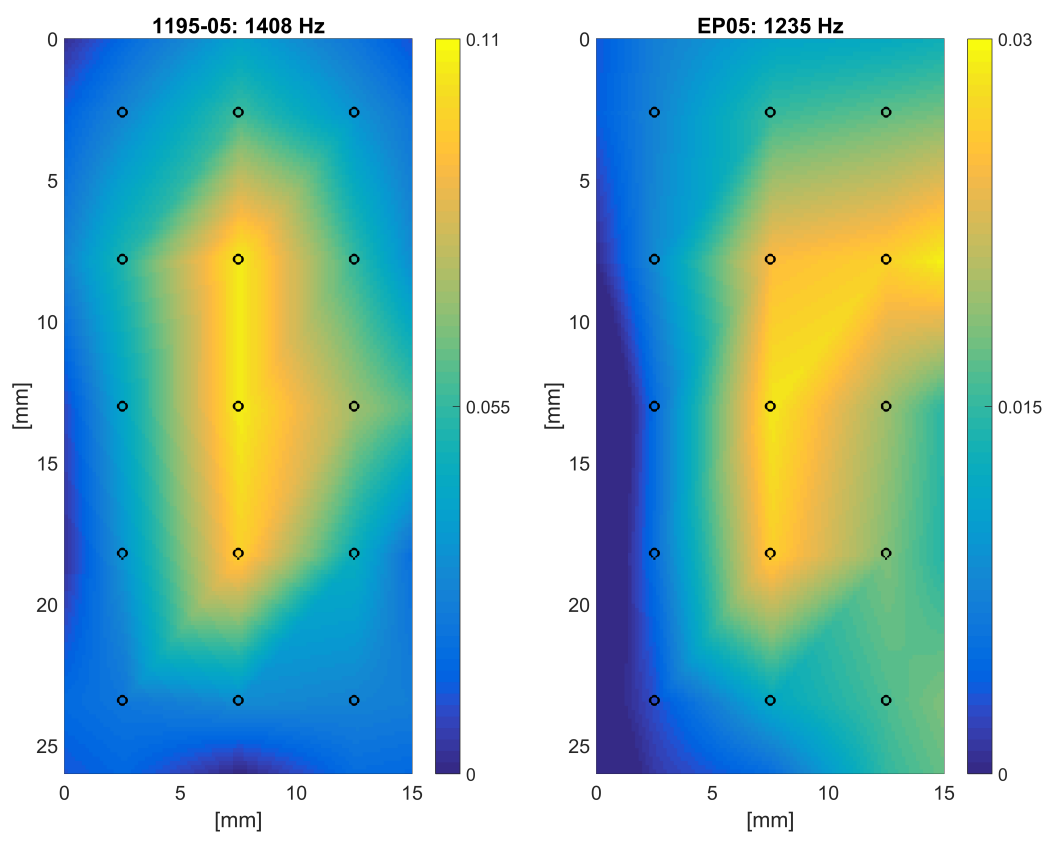

Figure 13. Transfer function between incident wave and vibrometer reading (velocity/deflection signal) for 1195-05 material: $1408 \mathrm{~Hz}$ and for EP-05 material: $1235 \mathrm{~Hz}$.

a fundamental mode shape occurs, with some artifacts on the upper left border for EP-05. Note, the very small values for the transfer function for EP-05, implying small velocity amplitudes and deflections of the vibration. By comparison of liner dissipation curve and frequencies of vibrations it turns out, that both are not connected. The dissipation maxima are mainly determined by the liner cavity structure, while the vibration of flexible walls are scarcely excited. Certainly, the higher stiffness of the rather thick flexible walls $(0.5 \mathrm{~mm})$ inhibits larger deflections.

A further quantity which can be derived from measurements in setup- 2 is the reflection coefficient of the liner cell wall samples. The ratio between reflected wave $p^{-}$and incident wave $p^{+}$should yield comparable information to the reflection coefficient of setup-1. In accordance with the above reasoning, that a vibrating structure dissipates and helps to transmit acoustic energy, a decrease in reflection coefficient should indicate a significant vibration. Figure 14 shows the reflection coefficients calculated from setup-2.

Frequencies with reflection coefficient minima coincide in part with results from setup- 1 and in most cases with the vibration maxima derived through the transfer functions. A detailed comparison of frequencies is given below in Table 1. 

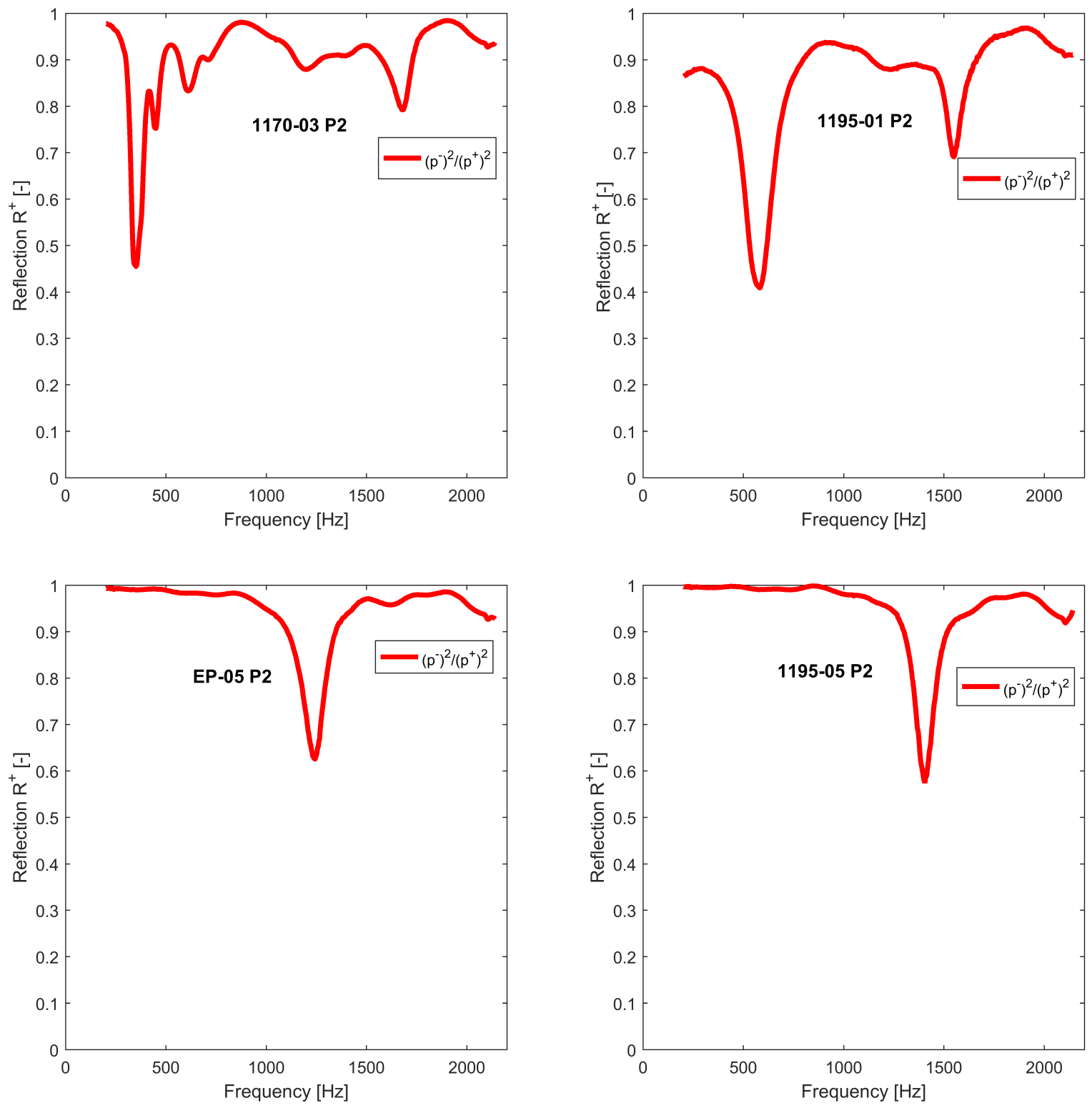

Figure 14. Reflection coefficientsfor setup-2. 


\section{Comparison of Measurements}

Table 1 summarizes the frequencies of vibration identified by the various measurements and different methods. Bold numbers mark major peaks, which are easily idetified when looking at respective plots. Smaller local maxima or minima, in some cases "bumps" which can only be identified when comparing with values of neigbouring frequencies are listed too (regular font). As already explained in previous sections, there is no single measurement procedure which perfectly predicts the specific features or frequencies of all liners with flexible structures. However, certain frequencies are well captured by different methods, as can be seen for the second frequency of material 1170-03.

\begin{tabular}{|c|c|c|c|c|c|}
\hline Material & Dissipation Liner & Setup-1 $T^{+}$ & Setup-1 $D_{\text {avg }}$ & Setup-2 (Vibro) & Setup-2 $R^{+}$ \\
\hline & Fig. 2 & Fig. 8 & Fig. 8 & Fig. 11-13 & Fig. 14 \\
\hline \multirow{5}{*}{$1170-03$} & $459 \mathrm{~Hz}$ (Bump) & $510 \mathrm{~Hz}$ & $408 \mathrm{~Hz}$ & $378 \mathrm{~Hz}$ & $341 / 454 \mathrm{~Hz}$ \\
\hline & $612 \mathrm{~Hz}(\mathrm{Max}-1)$ & & & $635 \mathrm{~Hz}$ & $612 \mathrm{~Hz}$ \\
\hline & $969-1020 \mathrm{~Hz}($ Max-2) & $1173 \mathrm{~Hz}$ & $1122 \mathrm{~Hz}$ & $1161 \mathrm{~Hz}$ & $1205 \mathrm{~Hz}$ \\
\hline & $1326 \mathrm{~Hz}(\mathrm{Max}-3)$ & $1428 \mathrm{~Hz}$ & $1377 \mathrm{~Hz}$ & $1310 \mathrm{~Hz}$ & \\
\hline & & & & $1650 \mathrm{~Hz}$ & $1676 \mathrm{~Hz}$ \\
\hline \multirow{4}{*}{$1195-01$} & $561 \mathrm{~Hz}$ (Bump) & & & & $580 \mathrm{~Hz}$ \\
\hline & $714 \mathrm{~Hz}(\mathrm{Max})$ & $663 \mathrm{~Hz}$ & $612 \mathrm{~Hz}$ & $630 \mathrm{~Hz}$ & \\
\hline & 969/1071 Hz (Bump) & $1275 \mathrm{~Hz}$ & $1224 \mathrm{~Hz}$ & $1240 \mathrm{~Hz}$ & $1209 \mathrm{~Hz}$ \\
\hline & 1479-1530 Hz (Bump) & & & $1536 \mathrm{~Hz}$ & $1552 \mathrm{~Hz}$ \\
\hline $1195-05$ & 816-867 Hz (Max) & $1071 \mathrm{~Hz}$ & $1071 \mathrm{~Hz}$ & $1408 \mathrm{~Hz}$ & $1407 \mathrm{~Hz}$ \\
\hline EP-05 & $867-918 \mathrm{~Hz}($ Max) & $1020 \mathrm{~Hz}$ & $1020 \mathrm{~Hz}$ & $1235 \mathrm{~Hz}$ & $1240 \mathrm{~Hz}$ \\
\hline
\end{tabular}

Table 1. Maxima identified from dissipation measurements (full liner), transmission and dissipation for liner wall sample (setup-1), and obtained from transfer function of vibration velocity(ref incident wave, setup-2). The dominating peaks are printed in bold letters.

The TPU samples with smaller wall thickness (1195-01 and 1170-03), which exhibit a drastic change in dissipation characteristics for the full liner structure (compared to the "REFERENCE" structure, Figure 2), are subject to stronger vibrations. Frequencies and mode shapes of these vibration can - at least in part - be investigated using the simplified liner wall samples. The measurements made relate the observed vibrations to the additonal dissipation of acoustic energy. The material inherent damping seems to influcence the overall dissipation characteristics to a much higher degree than for the other two samples (EP-05, 1195-05). The latter two samples do exhibit vibration peaks in the considered range, but these vibrations do not influence the overall acoustic dissipation of a respective liner structure.

\section{Conclusions and Outlook}

Different measurements were made on simplified liner wall samples in order to investigate the vibrations (deflection, velocity, mode shapes) of the flexible wall elements of the FlexiS liner concept. Measurements of reflection, dissipation, and transmission of acoustic energy shed some light on the material-dependent vibrations. Additional measurements with a vibrometer revealed spatial modes of the vibrating structure of fundamental and first higher order. Thereby, a direct link between the vibrating structure and some acoustic chararcteristics of a respective liner structure could be established. A perfect agreement of frequencies was not expected due to variations between liner structures and the samples investigated. This includes also the different boundary conditions for the cell walls in the liner assembly compared to the test setup.

With the acquired data, some additional information regarding the actual deflection and associated phase information might be obtained. Future work needs to focus on the analytical and numerical simulation of the vibrating structures. The data obtained through the current study may provide first validation for models. However, a higher spatial resolution of the structural vibration, considering also variations in material properties and of the boundary conditions will be required to build up a sufficient data base for model validation. 


\section{Acknowledgments}

The authors would like to thank the colleagues from TU Dresden, who prepared the samples for the test in the framework of LuFo V-2 project LaKS ("Laermabsorbierende Kunststoff-Strukturen/Noise Absorbing Composite Structures").

\section{References}

${ }^{1}$ Knobloch, K., Enghardt, L., and Bake, F., "Helmholtz Resonator Liner with Flexible Walls," 24th AIAA/CEAS Aeroacoustics Conference, No. 2018-4102, Atlanta, Georgia, USA, June 2018.

${ }^{2}$ BASF, "Thermoplastic Polyurethane Elastomeres," Elastollan ${ }^{\circledR}$ - Material Properties, issued September 2017, accessed online 07 November 2017. 